\title{
вмJ Global Health How can we better integrate the prevention, treatment, control and elimination of neglected tropical diseases with other health interventions? A systematic review
}

\author{
Gift Treighcy Banda (D) , ${ }^{1,2}$ Kebede Deribe, ${ }^{1,3}$ Gail Davey ${ }^{1,3}$
}

\begin{abstract}
To cite: Banda GT, Deribe K, Davey G. How can we better integrate the prevention, treatment, control and elimination of neglected tropical diseases with other health interventions? A systematic review. BMJ Global Health 2021;6:e006968. doi:10.1136/ bmjgh-2021-006968
\end{abstract}

Handling editor Stephanie M Topp

Received 22 July 2021 Accepted 27 September 2021

Check for updates

(c) Author(s) (or their employer(s)) 2021. Re-use permitted under CC BY-NC. No commercial re-use. See rights and permissions. Published by BMJ

${ }^{1}$ Department of Global Health and Infection, Brighton and Sussex Medical School, Brighton, UK

${ }^{2}$ Mzimba District Hospital, Malawi Ministry of Health, Mzimba, Malawi

${ }^{3}$ School of Public Health, Addis Ababa University, Addis Ababa, Ethiopia

Correspondence to Gift Treighcy Banda: gtreighb@gmail.com

\section{ABSTRACT}

Background Globally, about 1.7 billion people living in poverty are affected by one or more of a group of disabling, disfiguring and poverty-promoting conditions known as neglected tropical diseases (NTDs). Major global health actors, like the WHO, have endorsed a shift from vertical to integrated NTD management.

Objective This systematic review aimed to evaluate how integration is being conducted and how we can improve it. Methods PubMed, Medline, Cochrane library, Web of Science, Trip, Embase, Global Health and Google Scholar were searched from 1 April to 22 July 2020 .We included peer-reviewed articles published between 1 January 2000 and 22 July 2020 in English.

Results Database searches produced 24565 studies, of which 35 articles met the inclusion criteria. Twenty of these articles were conducted in sub-Saharan Africa. Twenty articles were also published between 2015 and 2020. Literature revealed that NTDs have been integrated-among themselves; with water, sanitation and hygiene programmes; with vector control; with primary healthcare; with immunisation programmes; and with malaria management. Integrated mass drug administration for multiple NTDs was the most common method of integration. The three complex, yet common characteristics of successful integration were good governance, adequate financing and total community engagement. Conclusion The dataset identified integrated management of NTDs to be cost effective and potentially to increase treatment coverage. However, the identified modes of integration are not exclusive and are limited by the available literature. Nonetheless, integration should urgently be implemented, while considering the programmatic and sociopolitical context.

PROSPERO registration number The study protocol was registered with PROSPER0 number, CRD42020167358.

\section{INTRODUCTION}

Neglected tropical diseases (NTDs) affect about 1.7 billion people globally. ${ }^{1}$ The disease burden of NTDs disproportionately affects those in low and middle-income countries.

\section{Key questions}

What is already known?

- Global health actors have been advocating for integrated management of neglected tropical diseases. Integrated management of diseases is said to be cost effective and improves the quality of care being delivered.

What are the new findings?

- Neglected tropical diseases (NTDs) have been integrated with other NTDs; with water, sanitation and hygiene services; immunisation programs; with integrated vector control; with primary healthcare; and with malaria. Community engagement, intersectoral and multidisciplinary collaborations as well as adequate funding and political will have been some of the key facilitators to successful integration.

- Unfavourable policy environment, difference in target groups and inadequate resourcing were found to be barriers for successful integration.

What do the new findings imply?

- Knowing what facilitates a successful integration program will enlighten policymaker. The results from this review provides data that can be used to inform decision makers on how to better implement integrated NTD management.

- Deliberate and systematic planning and efforts are required to achieve an integrated NTD programme streamlined with the broader health system to achieve the NTD road map 2021-2030 goals.

A variable number of conditions have been classified as NTDs by different organisations including the US' Centres for Disease Control, the WHO and other organisations. ${ }^{23}$ Twenty diseases were included in the WHO road map 2021-2030 to prevent, control, eliminate or eradicate NTDs, following gradual additions to the WHO NTD list between 2013 and 2019. ${ }^{4}$ Many of these diseases can be controlled, and even eliminated through mass drug 
administration (MDA), vector control and/or improved water, sanitation and hygiene (WASH) ${ }^{5}$ Sustained reduction in NTD-related mortality and morbidity requires a multisectoral and interdisciplinary approach. This includes health promotion, disease prevention, mobilising and empowering human resources, and strengthening service delivery through primary healthcare. ${ }^{3}$

Traditionally, there has been a disease-specific approach to tackling NTDs, through community-based mass campaigns and clinical management. ${ }^{6}$ For example, soil transmitted helminths ( $\mathrm{STH})$ and schistosomiasis are managed through MDA as preventive chemotherapy. ${ }^{7}$ Due to geographical overlap, limited resources and changing environments, global health actors have been advocating for integrated disease management. To increase awareness, upgrade treatment coverage and improve patient outcomes, the WHO has suggested that stakeholders should improve intersectoral coordination (eg, with WASH and tropical diseases research and training); integration across diseases and continued improvements to the medicine and resources supply chain. ${ }^{89}$

The new 2021-2030 WHO roadmap emphasises integrated NTD management to sustain the efforts made over previous years under the theme 'Ending the neglect to attain the Sustainable Development Goals' (SDGs). The WHO, policy-makers and academics have also suggested that endemic countries should integrate NTD management strategies using existing resources and into existing health interventions, since many of these diseases are coendemic. The WHO recommended the integration of NTDs into well-established public health programmes such as HIV/AIDS, malaria, maternal and child health and WASH programmes, or within NTDs (for example those that manifest as skin conditions-skin NTDs). ${ }^{10}$ Meanwhile, the UN has been advocating for Universal Health Coverage (UHC), in which communities are able to 'use the promotive, preventive, curative, rehabilitative, and palliative health services they need, of sufficient quality to be effective, while also ensuring that the use of these services does not expose the user to financial hardships'. ${ }^{11}$ The SDGs are robust in recognising the relationship between poverty, politics and health. Thus, they acknowledge the importance of controlling NTDs while conceptualising the main mission of UHC, which is 'leaving no one behind'. ${ }^{7}$ SDG 3 aims to achieve health equity through ensuring a healthy life and promoting well-being for all people. ${ }^{12}$ Tackling these neglected diseases is vital in health and development because UHC cannot be achieved if marginalised communities are still suffering. It is also a major step to reducing global poverty. Bangert et al noted how the integrated management of NTDs can help achieve several of the SDGs and vice versa. ${ }^{13}$ They indicated that integrated strategies to combat NTDs can boost global partnerships (SDG17), increase economic growth (SDG8) and generally reduce inequality (SDG10).

For the purpose of this systematic review, integration has been defined as the process by which disease control activities are functionally merged or coordinated within multifunctional healthcare delivery. ${ }^{6}$ Thus, healthcare systems are connected or combined with existing human services aimed at improving health outcomes. Integration is cost effective by reducing cost of delivery while minimising duplication of effort and resources in an already limited resource setting. ${ }^{14}$ Integrated disease management approaches are also said to help combat felt and experienced stigma caused by visible disability or disfiguring diseases such as Leprosy. ${ }^{15}{ }^{16}$ Integration of disease management with existing health interventions can improve the quality of care being delivered. ${ }^{4}$

Integrated disease interventions have been implemented in three ways to manage both communicable and non-communicable diseases: integration of similar diseases in the same category for example, NCDs; integration of diseases with existing programmes that are not necessarily in the same category for example, malaria and HIV; and integrating at a systems level, such as primary healthcare and procurement and supply management. ${ }^{1718}$ After multiple failed attempts to control malaria through vertical approaches, success has been shown through multiple integrations including with maternal health and procurement and supply. ${ }^{19}{ }^{20}$ There has been an increase in research papers on integrated management of NTDs, nevertheless, it is still uncertain which components of these interventions have contributed to the effectiveness or sustainability of outcomes. This systematic review summarises the approaches taken to integration of NTD programmes, characteristics and outcomes of successful integration.

\section{METHODS}

We conducted a systematic review of literature following a protocol that was registered with PROSPERO, registration CRD42020167358 which can be accessed from https://www.crd.york.ac.uk/prospero/display_record. php?ID=CRD42020167358. We reported following the Preferred Reporting Items for Systematic Reviews and Meta-Analysis. Between 1 April and 22 July 2020, we searched: Trip, Embase (Excerpta Medica dataBASE), Medline, PubMed, Cochrane library, Global Health and Web of Science for articles published between 1 January 2000 and 22 July 2020. We included peer reviewed articles that covered: preventing, improving the diagnosis of, treating, managing and monitoring NTDs; health promotion against NTDs; treatment compliance for NTDs and healthcare delivery and processes related to NTDs. Grey literature and articles that could not be Google translated into English were excluded.

We created a 22-disease list which was considered for this systematic review. Our search used a combination of population, intervention, comparison, outcome and advanced searches, which included exploded medical subject heading terms. Some search terms were truncated to capture various word endings (such as 'integration' and 'integrated'). When searching, methodological, 
geographical and language filters were not applied. Key words were searched in combination with individual diseases using the Boolean operators AND and OR (online supplemental appendix 1). Titles and abstracts were reviewed, and only relevant papers had their full papers retrieved. For articles whose full text was not available online, the corresponding authors were emailed for further details. If the author did not respond, the article was excluded from the study.

\section{Study selection}

We included primary studies that were in or could be Google translated into English. We disregarded articles that did not report on the integrated management of an NTD. The authors GTB and KD checked relevant articles.

The quality of studies was assessed using the NewcastleOttawa Scale (NOS).The NOS is a tool used to score non-randomised studies for systematic reviews and metaanalysis which assesses participant selection, comparability and results/outcomes. ${ }^{21}$ NOS follows a star rating. Poor quality studies scored 0 or 1 star across the assessed domains, while good quality studies scored a minimum of 2 stars across all domains. The critical appraisal skills programme ${ }^{22}$ was used in place of the NOS for primary studies. The quality appraisal tools were copied into a table then scored and marked for each study.

After searching the databases, the references were exported to EndNoteX7 and Mendeley. We created a Microsoft excel data extraction form and recorded the following information: author, title, year of study and publication, country of study, study population, diseases of study, mode of integration, outcome and sponsors. Data were extracted by GTB and reviewed by all authors. We undertook a narrative synthesis of the extracted data.

\section{ETHICAL CONSIDERATION}

This study followed the registered protocol.

\section{Patient and public involvement}

Neither patients nor the public were involved in the study design and article selection.

\section{RESULTS}

Our keyword search yielded 24565 studies (figure 1). After screening through titles, abstracts, and full texts, 35 articles met the inclusion criteria (table 1). Twenty-one studies were conducted in sub-Saharan Africa (AFR), and 20 papers were published between 2015 and 2020. Most articles reported integrated management of STH $(n=20)$. None of the articles evaluated the integration of the management or control of snakebite envenoming, sleeping sickness, mycetoma, leishmaniasis, chikungunya, dengue fever, echinococcosis, fascioliasis or dracunculiasis. Overall, the included studies had fair-good methodological quality, with some studies providing insufficient

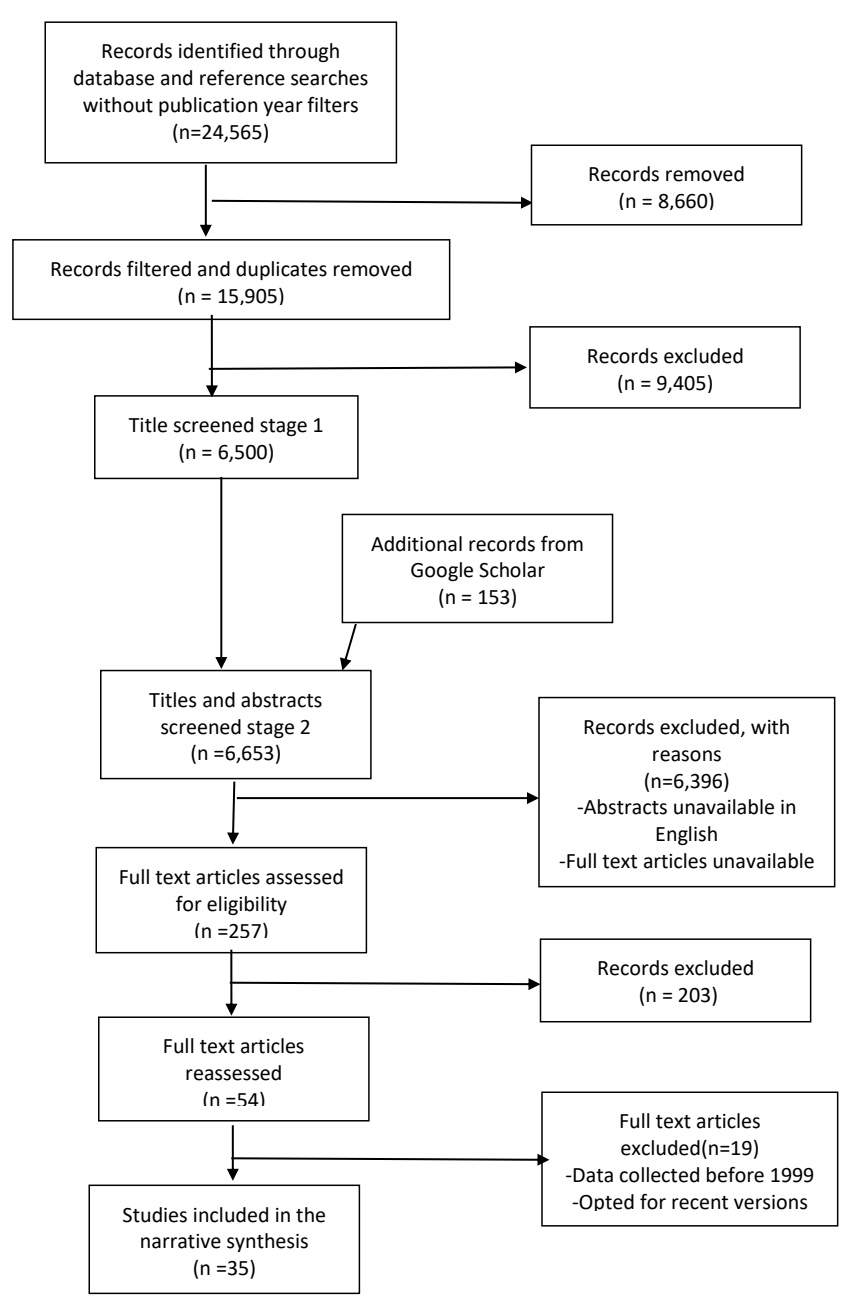

Figure 1 Preferred Reporting Items for Systematic Reviews and Meta-Analyses study flow diagram.

information on outcome measures online supplemental appendix 2.

\section{Identified integration modes}

In these studies, six different modes of integration were identified; with other NTDs $(n=21)$; with WASH services $(n=7)$; with immunisation $(n=3)$; with integrated vector control $(n=4)$; with primary healthcare $(n=4)$; and with malaria $(n=6)$. It is important to note that some studies integrated multiple strategies. For example, Knopp et $a l$ conducted an open trial on the control of urogenital schistosomiasis with MDA, behaviour change facilitated by community education and vector control of the snails in Zanzibar. ${ }^{23}$ They noted that neither communities that received MDA and behaviour change nor those that received MDA and snail control showed any significant difference in prevalence compared with the communities that only received MDA. ${ }^{23}$ One of the studies integrated MDA for STH, lymphatic filariasis (LF) and onchocerciasis with primary healthcare in Mali. ${ }^{24}$ They integrated the training of health workers for screening and referral where necessary. The significant gain of the integrated NTD programme was the scale-up of LF MDA which 


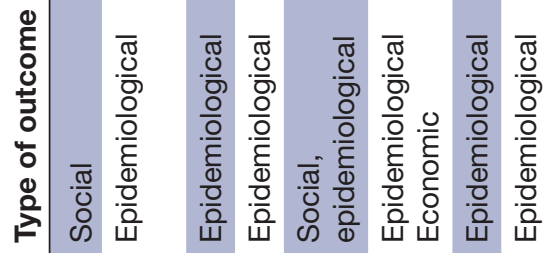

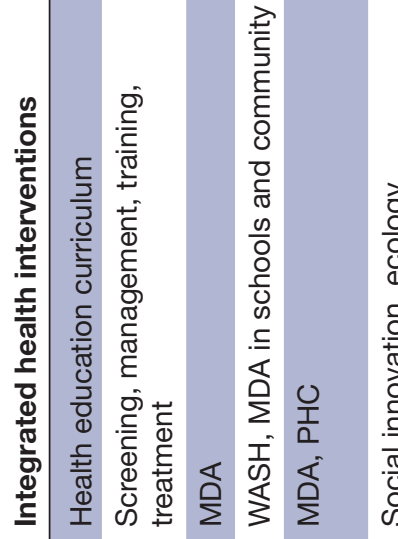

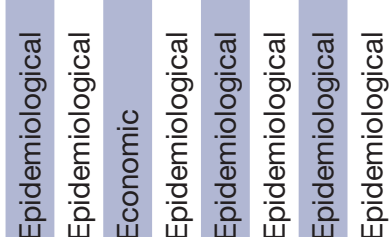

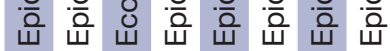

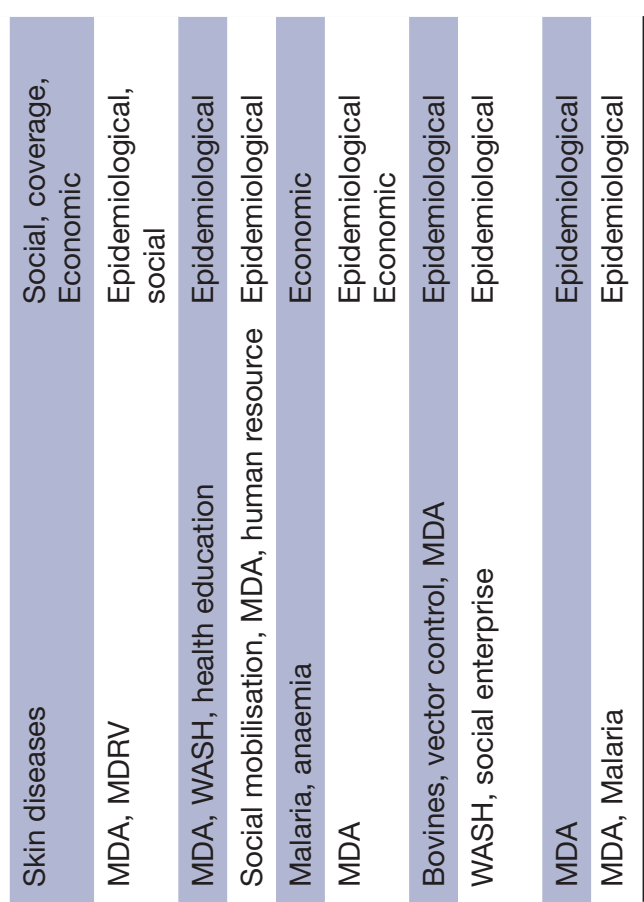

I

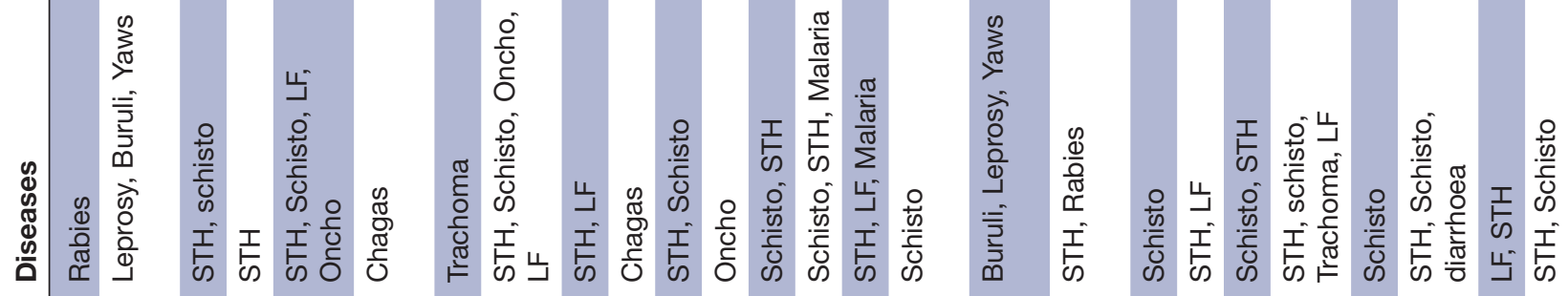

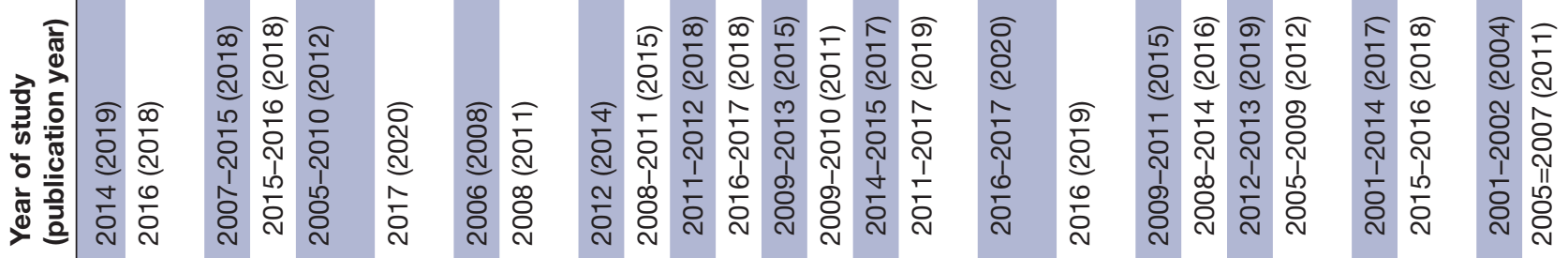
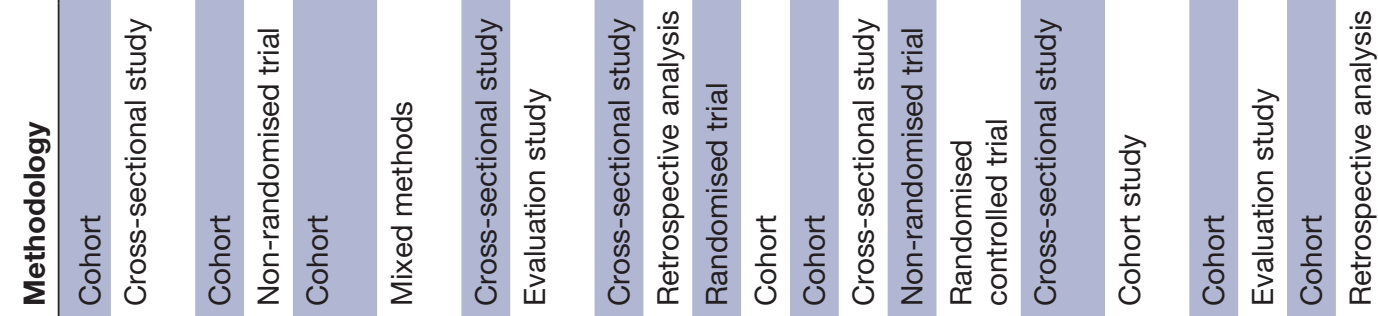

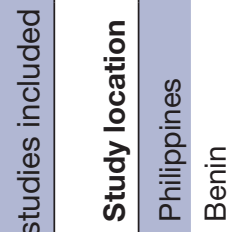

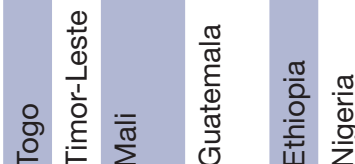

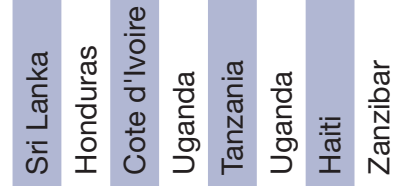

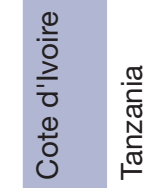

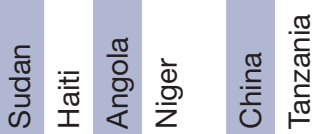

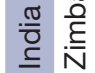

ले

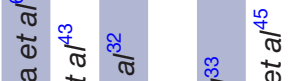

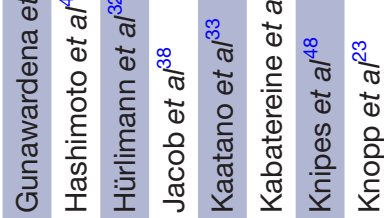

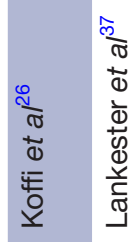

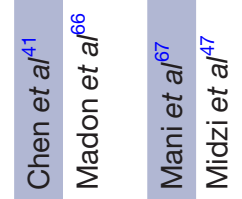

욱

웅

N

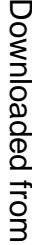

急

음

을.

을

임

을.

N

స్త్ర

ए

$\stackrel{0}{ }$

$\stackrel{\Phi}{\stackrel{1}{\longrightarrow}}$

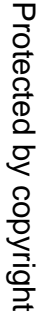




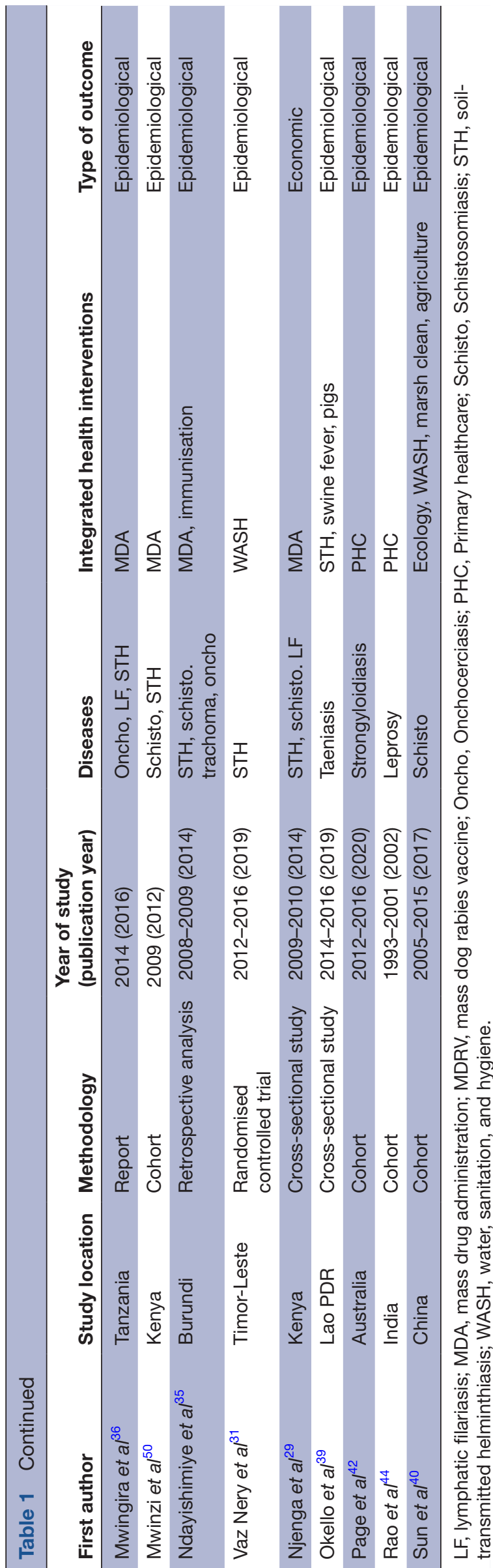

achieved full national geographic coverage in 2009. It is uncertain whether this intervention was cost effective or not, as the amount of money spent did not represent the full cost of logistics or staff salaries. For studies that integrated multiple strategies, only one method of integration has been chosen as a subheading for integration strategies.

\section{With other NTDs}

Skin-NTDs such as Buruli ulcer and yaws have been integrated with each other for diagnosis and treatment, ${ }^{2526}$ as well as other NTDs. Koffi et al trained nurses and community health workers together, in Côte d'Ivoire, in basic dermatology and disease management. Afterwards, health workers were more willing to implement integrated interventions to manage leprosy, Buruli ulcer and yaws than individual disease management, but they required capacity building and resources. ${ }^{26}$ Barogui et al conducted a similar study in Benin but used both health workers and community volunteers following the World Health Assembly recommendations. ${ }^{25}$ These allowed for continuous monitoring and sustainability of the programme. Both these studies trained nurses and peripheral health workers to be able to detect and treat skin conditions, with a focus on yaws, Buruli ulcer and leprosy. The combined training allowed the detection of other NTDs that were not targeted such as LF. Investigators discovered that health workers and volunteers were able to detect, manage and refer other skin conditions including scabies ${ }^{26}$ and $\mathrm{LF}^{25}$

Integrated MDA for two or more NTDs was also noted (table 2). A study in Mali looked at the integration of schistosomiasis, STH, LF and onchocerciasis MDA. ${ }^{24}$ The team distributed drugs for either two diseases, or all four diseases, depending on the disease mapping results. They reported an increase in coverage of up to $100 \%$ for LF drugs but did not indicate a baseline coverage. Bronzan et $a l^{27}$ reported no changes to treatment coverage but a significant reduction in the prevalence of schistosomiasis and STH after they integrated MDA among schoolchildren aged 6-10 years old in Togo. Out of these studies, two studies reported no changes in coverage of MDA or prevalence of $\mathrm{STH}^{28}{ }^{29}$; however, one study reported a reduction in the prevalence of schistosomiasis only. ${ }^{29}$ Evans $e t a l,{ }^{30}$ and Dembélé $e t a l^{24}$ reported 'minor' adverse reaction to their drug combinations while Lemos et al did not indicate the intensity of the adverse reaction which were experienced by $52 \%$ of their participants. ${ }^{28}$

\section{With WASH programmes}

WASH programmes are often categorised into those addressing quality and quantity of water, storage of water, hygiene and sanitation. Clarke et al piloted a study which was later altered and conducted by Nery $e t a l$. The studies combined school-based interventions and surveys with community-targeted projects in Timor-Leste. Nery et al improved school sanitation by incorporating hygiene education into school programmes and construction 
Table 2 Summary of studies that integrated mass drug administration

\begin{tabular}{|c|c|c|c|c|}
\hline Study author & Diseases & $\begin{array}{l}\text { Who distributed } \\
\text { the drugs }\end{array}$ & $\begin{array}{l}\text { Who managed } \\
\text { the programme }\end{array}$ & Outcome \\
\hline Lemos et $a l^{28}$ & $\begin{array}{l}\text { Schistosomiasis } \\
\text { STH } \\
\text { Malaria } \\
\text { Anaemia }\end{array}$ & $\begin{array}{l}\text { Centro de } \\
\text { Investigação em } \\
\text { Saúde de Angola } \\
\text { lab workers }\end{array}$ & $\begin{array}{l}\text { Government and } \\
\text { researchers }\end{array}$ & $\begin{array}{l}\text { All anaemia cases were associated } \\
\text { with schistosomiasis }-13.5 \% \text { reduction } \\
\text { in schistosomiasis infections with no } \\
\text { significant changes in anaemia }\end{array}$ \\
\hline Lemoine et $a l^{51}$ & $\begin{array}{l}\text { LF } \\
\text { STH }\end{array}$ & $\begin{array}{l}\text { Community drug } \\
\text { distributors, }\end{array}$ & $\begin{array}{l}\text { ENVISION } \\
\text { project }\end{array}$ & $100 \%$ coverage from as low as $30 \%$ \\
\hline Njenga et $\left.a\right|^{29}$ & $\begin{array}{l}\text { Hookworm (STH) } \\
\text { Urogenital } \\
\text { Schistosomiasis }\end{array}$ & $\begin{array}{l}\text { Trained } \\
\text { schoolteachers }\end{array}$ & Authors & $\begin{array}{l}\text { Did not change both the overall prevalence } \\
\text { and intensity of hookworm infection. }\end{array}$ \\
\hline $\begin{array}{l}\text { Ndayishimiye et } \\
a^{35}\end{array}$ & $\begin{array}{l}\text { STH } \\
\text { Schistosomiasis } \\
\text { Onchocerciasis } \\
\text { Trachoma }\end{array}$ & $\begin{array}{l}\text { Community drug } \\
\text { distributors and } \\
\text { community health } \\
\text { workers }\end{array}$ & $\begin{array}{l}\text { Ministry of } \\
\text { Health }\end{array}$ & $\begin{array}{l}\text { Increase in total population covered from } 3 \\
209521 \text { in } 2007 \text { to } 4179495 \text { in } 2010\end{array}$ \\
\hline Leslie et $a l^{65}$ & $\begin{array}{l}\text { STH } \\
\text { Schistosomiasis } \\
\text { LF } \\
\text { Trachoma }\end{array}$ & - & Government & $\begin{array}{l}\text { Integrated programme cost } 21 \% \text { less than } \\
\text { vertical programmes }\end{array}$ \\
\hline Evans et $a l^{30}$ & $\begin{array}{l}\text { STH } \\
\text { Schistosomiasis } \\
\text { Onchocerciasis } \\
\text { LF }\end{array}$ & $\begin{array}{l}\text { Community drug } \\
\text { distributors }\end{array}$ & $\begin{array}{l}\text { Ministry of } \\
\text { Health }\end{array}$ & $\begin{array}{l}98 \%-100 \% \text { coverage, reduction in cost by } \\
41.1 \%\end{array}$ \\
\hline Mwinzi et $a l^{50}$ & $\begin{array}{l}\text { STH } \\
\text { Schistosomiasis }\end{array}$ & $\begin{array}{l}\text { Community drug } \\
\text { distributors }\end{array}$ & $\begin{array}{l}\text { Ministry of } \\
\text { Health }\end{array}$ & $\begin{array}{l}52.3 \% \text { to } 91.9 \% \text { coverage with a } 69 \% \\
\text { prevalence reduction for hookworm }\end{array}$ \\
\hline Mani et al ${ }^{67}$ & $\begin{array}{l}\text { STH } \\
\text { LF }\end{array}$ & $\begin{array}{l}\text { Community health } \\
\text { workers }\end{array}$ & - & $\begin{array}{l}77 \% \text { prevalence reduction compared } 15 \% \\
\text { reduction for single drug ( } 3 \times \text { increase in } \\
\text { control) }\end{array}$ \\
\hline Bronzan et $\left.a\right|^{27}$ & $\begin{array}{l}\text { STH } \\
\text { Schistosomiasis }\end{array}$ & - & $\begin{array}{l}\text { Ministry of } \\
\text { Health }\end{array}$ & $\begin{array}{l}\text { Decrease in STH prevalence from } 31.1 \% \text { in } \\
2009 \text { to } 11 \% \text { in } 2015 \\
\text {-Schisto } 23.5 \% \text { to } 5 \%\end{array}$ \\
\hline
\end{tabular}

'-' means not clear.

LF, lymphatic filariasis; STH, soil transmitted helminths.

of concrete-lined pit latrines for both students and teachers. ${ }^{31}$ In the communities, they constructed taps, and encouraged a total sanitation approach. This saw an increase in latrine usage from $65.9 \%$ to $84.9 \%$, an increase in reported hand-washing practices and an STH prevalence reduction of $88.2 \%$ (95\% CI 70.2 to 100.0 ). ${ }^{31}$ The intervention group also saw a reduction of the odds of STH by $57 \%$ compared with the control group (which only received school-based intervention). They also noted that half of the study population were adults, $40 \%$ of whom had never received formal education.

Hürlimann $e t a \hat{l}^{2}$ conducted a controlled trial in Cote d'Ivoire on the general population. They integrated the control of schistosomiasis and STH with community-led total sanitation (an expansion of the WASH programme that discourages open community defecation). They noted that the intervention reduced the prevalence of schistosomiasis but did not show any effect on the prevalence of STH. Interestingly, Nery et al also noticed that their WASH and MDA programme for STH did not result in a significant change in the prevalence of the disease compared with MDA alone. ${ }^{31}$ Kaatano et al improved the quality of water that families used and tried to reduce their contact with lake water by constructing pump wells on Kome island in Tanzania. ${ }^{33}$ This was combined with health education and MDA. The intervention resulted in a reduction in the prevalence of schistosomiasis by $90.5 \%$ in children and $83 \%$ in adults. They also noted a STH reduction of $93.3 \%$ in children and $56.9 \%$ in adults. Similarly in Sudan, investigators constructed a filtered drinking water supply to supplement MDA. ${ }^{34}$ A clean water supply within the intervention village contributed significantly to the reduction in the prevalence of urogenital schistosomiasis (from $28.5 \%$ to $13.5 \%$ ), in comparison to the control village.

\section{With immunisation programs}

The Burundian Ministry of Health ran a campaign to incorporate MDA for STH, schistosomiasis, onchocerciasis and trachoma. ${ }^{35}$ Treatment for STH was incorporated countrywide, while MDA for the other diseases was only distributed in endemic districts. They integrated these with immunisation programmes for children aged up to 59 months, antenatal clinics for pregnant women, 
primary schools and in communities. The drugs were distributed by community drug distributers and local health facility staff. For trachoma, community health workers were the only ones that assessed, diagnosed, confirmed and provided medication. For all diseases, reported coverage in children was $95 \%-100 \% .{ }^{35}$ Mwingira et al integrated NTD programmes (MDA) with the measles and rubella immunisation programme (MR) in Tanzania. ${ }^{36}$ The MR vaccines were delivered to children aged 9 months to 15 years (to accommodate catch-up doses) together with mebendazole for those aged 1-5 years. The MDA was for LF, onchocerciasis and STH for those aged over 5 in the same geographical area. They integrated the two programmes by planning, sensitising the community, drug distribution (and vaccination) and postadministration evaluation. The vaccination coverage remained at $97 \%$ while the MDA coverage increased from $86 \%$ to $93 \%$. Lankester et al also integrated the vaccination of dogs with the MDA of STH drugs in Tanzania. ${ }^{37}$ They followed three cohorts of community dwellers. Cohort A received combined deworming and mass dog rabies vaccine, cohort $\mathrm{B}$ received deworming drugs, and cohort $\mathrm{C}$ received the rabies vaccine only. The group that received the combined delivery of MDA and MRDV saved 32 min walking time. This group also experienced a 33\% lower cost per deworming dose and a $16 \%$ lower cost per rabies vaccination. It was unclear how the savings came about, but could be from cutting logistical costs. ${ }^{37}$

\section{With vector control programmes}

In Uganda, Jacob et $a l^{88}$ supplemented the MDA programme for onchocerciasis with community-directed slashing and clearing of vegetation which served as potential breeding areas. This clearing led to $89 \%-99 \%$ decline in vector biting rates. However, this study did not record the effect of this, combined with the MDA on disease prevalence. Okello et al also reported a $63 \%$ reduction in disability-adjusted life years (DALYs) after integrating human-alone Taeniasis management with that of STH and Taeniasis in humans and pigs in northern Lao. ${ }^{39}$ They compared the cost (in monetary value and DALYs) of control of Taeniasis alone or with three possible combinations. Thus, Taeniasis/cysticercosis in the human population (the base case), Taenia solium/cysticercosis and STH in the human population; T. solium/cysticercosis alone in the human and pig population (which also acts a vector of transmission to humans); T. solium/cysticercosis in the pig population and STH in humans; and finally T. solium/cysticercosis, STH and classical swine fever in humans and pigs. They estimated US\$214 and US\$93 per DALY averted in both pigs and humans, respectively. ${ }^{39}$

China has moved the control of schistosomiasis from disease-based to environmental through improvement of marshland areas ${ }^{40} 41$ which has taken 10-15 years to achieve. The integrated environmental improvement project included clearing marshland, building of boat factories and burying of soil with contaminated snails.
They also introduced fish in some of the marshland areas to serve as biological control agents. They reported a reduction in infection rates in both humans and cows to 0 in 2015 , from 0.4 in humans and 1.3 in livestock in 2003. They also reported a reduction in human cases from 61 in 2004 to 0 from 2005 to $2015 .^{40}$ The multisectoral involvement showed the feasibility of eliminating schistosomiasis.

\section{With primary healthcare}

Page et al integrated screening for strongyloidiasis within primary healthcare among aboriginal Australians. ${ }^{42}$ Primary health providers incorporated screening for strongyloidiasis with routine medical check-ups and noticed an increase in the proportion screened from $40.3 \%$ to $81.9 \% .^{42}$ In Honduras, Hashimoto et at $t^{43}$ integrated Chagas disease vector control into routine disease prevention at health centres. Health centre staff promoted bug searching and vector reporting and supplied educational materials for community dwellers. They noticed a reduction in DALYs by $92 \%$, saved US $\$ 65$ 876 and improved disease surveillance from about $46 \%$ to $84 \%$. A similar approach was applied in Mali where community health workers are responsible for distribution of drugs and recruiting/ training community volunteers to work as drug distributors. ${ }^{24}$ They also reported an increase in coverage for all diseases that were included in the MDA (LF, onchocerciasis, schistosomiasis, STH and trachoma) with a coverage between $76 \%$ and $100 \%$ from as low as $39.6 \% .{ }^{24}$ After noticing that vertical approaches to the management of leprosy were no longer cost effective, the Indian government decided to integrate leprosy management into primary healthcare. ${ }^{44}$ They assigned one leprosy worker at a primary health centre who was a case detector and followed up patients through the same primary health centre. They noted an increase in older patients who presented themselves to the health facility. ${ }^{44}$

\section{With malaria programs}

Malaria was the only priority disease that was integrated with NTD interventions in the studies included. ${ }^{45}{ }^{46} \mathrm{In}$ Uganda, investigators integrated the management of schistosomiasis, STH and malaria to evaluate the prevalence of each disease, as well as the prevalence of coinfection. ${ }^{45}$ They recruited and trained community medicine distributors, who also acted as community mobilisers. All the participants received PZQ (Praziquantel) and ALB (Albendazole) and only those who tested positive for malaria through microscopy were given Artemisininbased combined therapy. ${ }^{45}$ Overall prevalence of coinfection with malaria and STH was $13.8 \% .{ }^{45}$ Prompt malaria tests and treatment with school-based MDA for STH and schistosomiasis were conducted every 6 months for a year and a half in Zimbabwe. STH and malaria coinfections reduced by $68 \%$, schistosomiasis and malaria by $84 \%$, and schistosomiasis and STH by $90.7 \% .{ }^{47}$ The final study was a pilot study conducted to assess the feasibility of an integrated LF-STH and malaria survey in Haiti. ${ }^{48}$ Surveys 
for LF-malaria were administered in 12 communities, for LF only in one and for LF-malaria and STH in one. Each survey was administered by a different team but at the same time, with the LF-STH-malaria team spending five more days collecting samples than the LF-STH and LF-malaria teams. The cost of the LF-STH-malaria evaluation was $49 \%$ higher than the cost for the LF-only evaluation, but did not disclose how much the malaria-only cost. $^{48}$

\section{Health education programs}

An integral part of the intervention studies included in this systematic review was health education. Health education was conducted alongside MDA, ${ }^{23} 3334$ immunisation and WASH programmes. ${ }^{32} 34$ Health education included education on health promotion, disease progression and signs and symptoms. Amparo et al integrated the rabies elimination programme into all elementary school curricula in The Philippines. ${ }^{49}$ Information on rabies, animal bite prevention, bite management and responsible pet ownership were strategically designed to be incorporated into all lessons including Maths, English, Filipino and the arts. Rabies knowledge among students significantly improved and bite incidents almost halved. From the included studies, three overarching themes which influenced the success or challenged the integrated interventions are collated within the label of barriers and facilitators.

\section{Facilitators of successful integration}

This review has shown that barriers and facilitators to integration cannot be viewed independently but as interlinked. For most studies, intersectoral and multidisciplinary collaborations, political will and community engagement facilitated the interventions (table 3 ). Community engagement was one of the most common themes that emerged from this review, especially for communitydirected interventions. ${ }^{42} 50$ Evans $e t \quad a l^{30}$ stated that community mobilisation and the usage of communitydirected distributors was an important factor in the integration of MDA for STH, schistosomiasis, onchocerciasis and LF. Likewise, Lemoine et $a \tilde{l}^{1}$ reported that community engagement was a key strategy to the success of their programme. This included selecting community members to train for the drug administration and education campaigns and ensuring community awareness of the programme. Effective communication was reported to increase community engagement and participation ${ }^{24}$ and brought a sense of programme ownership. ${ }^{30}$

\section{Barriers to integration}

Clarke et al failed to randomise their study groups because they were unable to find partners who would sponsor the study. ${ }^{52}$ This suggests that adequate funding is fundamental to high-quality studies and successful integrated programmes. Lack of community mobilisation was a threat to interventions as shown in Kenya where it led to a low treatment coverage of $46 \%$ for LF and schistosomiasis. ${ }^{29}$ Both studies that integrated the management of skin NTDs reported the lack of a dermatologist in their

Table 3 Factors that facilitated the integration of neglected tropical disease

\begin{tabular}{|c|c|c|c|c|c|}
\hline $\begin{array}{l}\text { Modes of } \\
\text { integration }\end{array}$ & Logistics & Good governance & Motivated actors & Policy & $\begin{array}{l}\text { Community } \\
\text { engagement }\end{array}$ \\
\hline With other NTDs & $\begin{array}{l}\text { Availability of public } \\
\text { health infrastructure } \\
64 \\
\text { Adequate funding }\end{array}$ & $\begin{array}{l}\text { Multilevel } \\
\text { stakeholders }^{435063}\end{array}$ & $\begin{array}{l}\text { Health workers } \\
\text { willingness to } \\
\text { provide integrated }^{\text {services }}{ }^{2526}\end{array}$ & & $\begin{array}{l}\text { Use of trusted } \\
\text { community health } \\
\text { workers }^{51} \\
\text { Community } \\
\text { sensitisation }^{242664} \\
\text { Health promotion } \\
\text { campaigns, }{ }^{23} 3334\end{array}$ \\
\hline $\begin{array}{l}\text { Water, sanitation } \\
\text { and hygiene } \\
\text { programmes }\end{array}$ & $\begin{array}{l}\text { Good coordination } \\
\text { between community } \\
\text { workers and } \\
\text { supervisors. }^{31}\end{array}$ & & & & $\begin{array}{l}\text { Community education } \\
\text { and sensitisation. }{ }^{32} 34\end{array}$ \\
\hline $\begin{array}{l}\text { Immunisation } \\
\text { programmes }\end{array}$ & & $\begin{array}{l}\text { Government } \\
\text { ownership }\end{array}$ & & $\begin{array}{l}\text { Policy } \\
\text { supporting } \\
\text { integrating } \\
\text { methods }\end{array}$ & $\begin{array}{l}\text { Timely communication } \\
3736\end{array}$ \\
\hline Vector control & & & & & $\begin{array}{l}\text { Community } \\
\text { sensitisation }^{38}\end{array}$ \\
\hline Primary healthcare & & $\begin{array}{l}\text { National strategic } \\
\text { plan ownership }\end{array}$ & $\begin{array}{l}\text { Willingness of } \\
\text { health workers }\end{array}$ & & \\
\hline $\begin{array}{l}\text { Malaria } \\
\text { programmes }\end{array}$ & & & $\begin{array}{l}\text { Use of trusted } \\
\text { community drug } \\
\text { distributors }^{45}\end{array}$ & & $\begin{array}{l}\text { Community } \\
\text { mobilisation and } \\
\text { sensitisation }^{45}\end{array}$ \\
\hline
\end{tabular}


study as a limitation. ${ }^{25} 26$ They also acknowledged the lack of medication or affordable drugs for the patients as being counterproductive to their efforts. ${ }^{26}$ Unfavourable national policies had a negative impact, as in the case of Zimbabwe, ${ }^{47}$ where only certified medical personnel are allowed to administer any form of medication, which limited MDA coverage. Similarly, Emerson et al successfully managed to conduct a survey on malaria and trachoma but noted that integrated management of these two diseases may not work. ${ }^{53}$ The policy for malaria net distribution is to give two nets per household and only requires one household member, while the trachoma management would need the entire household to be present.

\section{DISCUSSION}

This systematic review presented the available literature regarding the integration of NTD management with existing health interventions. The new 2021-2030 WHO roadmap emphasises that integrated NTD management to sustain reductions in NTD related mortality and morbidity, requires a multisectoral and interdisciplinary approach. ${ }^{10}$ We conducted a systematic review of 35 articles, predominantly from sub-Saharan Africa, to synthesise evidence of applying integrated management methods to NTDs. The literature has shown six dominant ways in which the integration of NTDs is applied in different policy settings and different sociocultural environments. These are with: other NTDs; WASH programmes; immunisation; integrated vector control; primary healthcare; and malaria.

We acknowledge these modes of integration are not exhaustive and are limited by the available literature. With the disfiguring and disabling nature of NTDs, we expected to find literature on integrated morbidity management as this is also a vital part of disease management. ${ }^{54}$ Alleviating the suffering caused by disease morbidity should also be a priority. The fact that most studies integrated the management of multiple NTDs supports that there is high level of coendemicity and geographical overlap, as reported by Hotez et $a l^{55}$ The study has also shown that interventions against similar diseases, such as skin NTDs, are relatively easy to integrate, as suggested by Foster. ${ }^{56}$ The skin-NTDs studies support Narain et al s observation that integrated disease management boosts the skills of the health workers. ${ }^{17}$ Molyneux and Nantulya advocated for linking malaria control programme to onchocerciasis, LF, schistosomiasis, measles and trachoma. ${ }^{57}$ This systematic review shows how this has been done for LF, STH and schistosomiasis. None of the literature covered integration of malaria with onchocerciasis nor trachoma. This may reflect a missed opportunity.

An important part of the integration, especially for school and community-based interventions, was health education. Health education integrated into school curricula has been shown to have a positive impact on children. Ejike et al modified the 'snakes and ladders' game to 'schisto and ladders' to educate students about schistosomiasis in Nigeria. ${ }^{58}$ The game included information on schistosomiasis transmission and safety of praziquantel. They noted a significant improvement in the knowledge of risk behaviours, prevention and control of schistosomiasis among the group that played schisto and ladders compared with the group that played the traditional snakes and ladders. This supports Amparo et al s approach of creatively integrating educational information into school-appropriate lessons and activities. ${ }^{49}$ It is imperative that the use of edutainment be incorporated in health promotion for school-going children.

Studies have shown that barriers to integrating disease management go beyond the availability of resources. The findings showed that community participation and adequate funding, as well as good governance (political will) are key to the success of integrated programmes. The three facilitators included interlinked subthemes. For example, good leadership and literacy can be associated with community engagement and communication. Good leadership and government ownership, combined with availability of resources and infrastructure that is, for referrals, are important when implementing integrated services. Governments should ensure that NTDs are integrated into national health plans and budgets for accountability and ensuring adequate funding. The findings showed that MDA programs that included health education and selected community members as drug distributors were successful. Parker and Allen ${ }^{59}$ noted that the success of MDA programs was also determined by the relationship between drug distributors and the study participants. Baker et al proposed that integrated programmes should continually be evaluated for impact as opposed to just 'pushing integration for the sake of integration'. ${ }^{60}$ This will limit duplication from other settings and ensure that challenges are being addressed. Health systems are not uniform, so the facilitators identified in this study should be analysed and adapted to specific country needs to ensure that strategies are impact oriented.

As discourse on how we can improve the integration of NTDs occurs, we should also look at how vertical approaches are impacting NTDs and how NTD control can impact other diseases. Indeed, health interventions targeting other diseases have positively impacted the control of NTDs, especially those with similar approaches. For example, the use of insecticidal nets to combat malaria in The Gambia led to the interruption of LF transmission since they have a common vector. ${ }^{61}$ Deliberate efforts to tackle NTDs can benefit from strengthened health systems while themselves contributing to improved health systems. National programme managers should consider ways in which NTDs can help with other disease burdens, especially those that may benefit from community health workers, water and sanitation and similar skilled workers. For example, community workers trained in active case-finding can be used to survey and monitor emerging diseases. 
The integration of NTDs within the general health system requires careful and deliberate planning. Countries should address this systematically. Leadership and governance of the health system at all levels should include NTDs. Countries should include NTD prevention and treatment services in their essential healthcare packages and universal healthcare benefit packages. The national health information system should also include NTD indicators and data collection systems to sustainably track progress and inform decision making at all levels. Integration of the NTD drug supply system within the national medical supplies and logistic system is required. Integration of NTDs with the training curriculum of health workforce is also required to ensure preservices training of health providers. To ensure a multisectoral response to NTD control, elimination and eradiation, it is critical to establish or strengthen multisectoral coordination body, engage other sectors such as education; WASH; gender and social inclusion; agriculture sector in the planning and financing of NTD interventions.

The impact of COVID-19 on health systems and the global economy also means that integrated delivery of health programmes is critical to optimally use the available budget. Integration will play an important role in leveraging resources in different health programmes and campaigns and provide services that attain the highest standards of public health measures against COVID-19, protecting both the public and those who deliver the services. Molyneux et al suggest that safe MDA distribution can mitigate the impact of COVID-19. ${ }^{62}$ In addition, integration will also improve adherence to COVID-19 prevention methods such as social distancing by reducing the number of contacts with community health workers and with the healthcare system.

\section{CONCLUSION}

The review identified six modes of integration. These are with: other NTDs; WASH programmes; immunisation, vector control; primary healthcare and malaria. The application of integrated management of diseases is context-dependent and may not be applied universally. Thus, the wider sociocultural and sociopolitical contexts must be analysed during the needs assessment stage. Despite this, these studies can serve as guidance for future integration studies. In these studies, three main themes that acted as barriers or facilitators to integration emerged. These included good governance, political will, funding and community engagement. This has shown that the effectiveness and success of such programmes go beyond the availability of resources.

Ultimately, integrated management of NTDs appears promising, with endemic countries producing research papers on how they are implementing strategies. With continual support and monitoring of progress by larger organisations such as the WHO, the accountability endemic countries have towards achieving their goals is likely to increase. The results from this review provide data that can be utilised to inform decision makers on how to better implement NTD management.

\section{Twitter Gift Treighcy Banda @GtreighB}

Contributors All authors contributed to this research. GTB and KD conceptualised the study and checked relevant studies. GTB prepared the manuscript, GD and KD added important intellectual input. All authors approved of the final manuscript. GTB acts as the guarantor on behlaf of all authors.

Funding KD is supported by the Wellcome Trust [grant number 201900/Z/16/Z] as part of his International Intermediate Fellowship.

Competing interests None declared.

Patient consent for publication Not applicable.

Provenance and peer review Not commissioned; externally peer reviewed.

Data availability statement № data are available.

Supplemental material This content has been supplied by the author(s). It has not been vetted by BMJ Publishing Group Limited (BMJ) and may not have been peer-reviewed. Any opinions or recommendations discussed are solely those of the author(s) and are not endorsed by BMJ. BMJ disclaims all liability and responsibility arising from any reliance placed on the content. Where the content includes any translated material, BMJ does not warrant the accuracy and reliability of the translations (including but not limited to local regulations, clinical guidelines, terminology, drug names and drug dosages), and is not responsible for any error and/or omissions arising from translation and adaptation or otherwise.

Open access This is an open access article distributed in accordance with the Creative Commons Attribution Non Commercial (CC BY-NC 4.0) license, which permits others to distribute, remix, adapt, build upon this work non-commercially, and license their derivative works on different terms, provided the original work is properly cited, appropriate credit is given, any changes made indicated, and the use is non-commercial. See: http://creativecommons.org/licenses/by-nc/4.0/.

\section{ORCID iD}

Gift Treighcy Banda http://orcid.org/0000-0002-7465-0730

\section{REFERENCES}

1 Uniting to Combat NTDs. Powering our partnership to fight all 20 neglected tropical diseases [Internet], 2018. Available: http:// unitingtocombatntds.org/resource/london-declaration [Accessed cited 2020 Aug 24].

2 CDC. Which diseases are considered neglected tropical diseases? [Internet. Centers for Disease Control and Prevention, 2019: 1. https://www.cdc.gov/globalhealth/ntd/diseases/index.html

3 WHO. The neglected tropical diseases: a rags-to- riches story: ten years in public health. World Health Organisation, 2017: 1-9. https:// www.who.int/publications/10-year-review/ntd/en/

4 WHO. Report of the who strategic and technical Advisory group for neglected tropical diseases. World Health Organization, 2019: 1. http://www.who.int/neglected_diseases/NTD_STAG_report_2015. pdf?ua $=1$

5 Mitra A, Mawson A. Neglected tropical diseases: epidemiology and global burden. Trop Med Infect Dis 2017;2:36.

6 Gyapong JO, Gyapong M, Yellu N, et al. Integration of control of neglected tropical diseases into health-care systems: challenges and opportunities. Lancet 2010;375:160-5.

7 Molyneux DH, Savioli L, Engels D. Neglected tropical diseases: progress towards addressing the chronic pandemic. Lancet 2017;389:312-25.

$8 \mathrm{WHO}$, Carter Center. Integrated control of the neglected tropical diseases: a neglected opportunity ripe for action. Atlanta, Georgia: World Health Organisation, 2008: 1-6. https://www.who.int/ neglected_diseases/WHO_HTM_NTD_2008.1_OK.pdf

9 WHO. Why are some tropical diseases called neglected [Internet]. World Health Organisation, 2019. Available: https://www.who.int/ neglected_diseases/en/ [Accessed 18 Apr 2020].

10 World Health Organisation. Ending the neglect to attain the Sustainable Development Goals - A road map for neglected tropical diseases 2021-2030. Geneva World Heal Organ [Internet], 2020. Available: http://apps.who.int/bookorders

11 Vega J. Universal health coverage: the post-2015 development agenda. Lancet 2013;381:179-80.

12 United Nations. A History of Universal Health Coverage in the UN [Internet]., 2019. Available: https://www.uhc2030.org/un-hlm-2019/ 
a-history-of-universal-health-coverage-in-the-un/ [Accessed 10 May 2020].

13 Bangert M, Molyneux DH, Lindsay SW, et al. The cross-cutting contribution of the end of neglected tropical diseases to the sustainable development goals. Infect Dis Poverty 2017;6:73.

14 Lamptey P, Dirks R, Torpey K. Discussion paper on how to promote the inclusion of the prevention and control of noncommunicable diseases within other programmatic areas 2016.

15 Molyneux DH, Hotez PJ, Fenwick A. "Rapid-impact interventions": how a policy of integrated control for Africa's neglected tropical diseases could benefit the poor. PLoS Med 2005;2:e336-70.

16 Kumar Gupta A. Integrated approach for leprosy elimination in India. J Dev Soc Transform 2004;1:31-6.

17 Narain JP. Integrating services for noncommunicable diseases prevention and control: use of primary health care approach. Indian $J$ Community Med 2011;36:67.

18 Banda GT. Integration of the management of NTDs with existing health interventions: research proposal. Brighton, 2020.

19 Mbonye AK, Hansen KS, Wamono F, et al. Integration of malaria and HIV/AIDS prevention services through the private sector in Uganda. Int Health 2010;2:52-8.

20 Schantz-Dunn J, Nour NM. Malaria and pregnancy: a global health perspective. Rev Obstet Gynecol 2009;2:186-92.

21 Wells G, O'Connell D, Peterson J. Newcastle-Ottawa quality assessment scale [Internet. Ottawa Hospital Research Institute, (OHRI), 2014: 2-4. http://www.ohri.ca/programs/clinical_ epidemiology/oxford.asp

22 Oxford Centre for Triple Value Healthcare. CASP Checklists - CASP - Critical Appraisal Skills Programme [Internet]., 2020. Available: https://casp-uk.net/casp-tools-checklists/

23 Knopp S, Person B, Ame SM, et al. Evaluation of integrated interventions layered on mass drug administration for urogenital schistosomiasis elimination: a cluster-randomised trial. Lancet Glob Health 2019;7:e1118-29.

24 Dembélé M, Bamani S, Dembélé R, et al. Implementing preventive chemotherapy through an integrated national neglected tropical disease control program in Mali. PLoS Negl Trop Dis 2012;6:e1574

25 Barogui YT, Diez G, Anagonou E, et al. Integrated approach in the control and management of skin neglected tropical diseases in Lalo, Benin. PLoS Negl Trop Dis 2018;12:e0006584.

26 Koffi AP, Yao TAK, Barogui YT, et al. Integrated approach in the control and management of skin neglected tropical diseases in three health districts of Côte d'Ivoire. BMC Public Health 2020;20:1-9.

27 Bronzan RN, Dorkenoo AM, Agbo YM, et al. Impact of communitybased integrated mass drug administration on schistosomiasis and soil-transmitted helminth prevalence in Togo. PLoS Negl Trop Dis 2018;12:e0006551.

28 Lemos M, Fançony C, Moura S, et al. Integrated communitybased intervention for urinary schistosomiasis and soil-transmitted helminthiasis in children from Caxito, Angola. Int Health 2020;12:86-94.

29 Njenga SM, Mutungi FM, Wamae C, et al. Once a year school-based deworming with praziquantel and albendazole combination may not be adequate for control of urogenital schistosomiasis and hookworm infection in Matuga district, Kwale County, Kenya. Parasit Vectors 2014;7:74-9.

30 Evans D, McFarland D, Adamani W, et al. Cost-Effectiveness of triple drug administration (TDA) with praziquantel, ivermectin and albendazole for the prevention of neglected tropical diseases in Nigeria. Ann Trop Med Parasitol 2011;105:537-47.

31 Vaz Nery S, Traub RJ, McCarthy JS, et al. Wash for worms: a clusterrandomized controlled trial of the impact of a community integrated water, sanitation, and hygiene and deworming intervention on soil-transmitted helminth infections. Am J Trop Med Hyg 2019;100:750-61.

32 Hürlimann E, Silué KD, Zouzou F, et al. Effect of an integrated intervention package of preventive chemotherapy, community-led total sanitation and health education on the prevalence of helminth and intestinal protozoa infections in Côte d'Ivoire. Parasit Vectors 2018;11:1-20.

33 Kaatano GM, Siza JE, Mwanga JR, et al. Integrated schistosomiasis and Soil-Transmitted helminthiasis control over five years on Kome Island, Tanzania. Korean J Parasitol 2015;53:535-43.

34 Lee $\mathrm{Y}-\mathrm{H}$, Jeong $\mathrm{HG}$, Kong $\mathrm{WH}$, et al. Reduction of urogenital schistosomiasis with an integrated control project in Sudan. PLOS Negl Trop Dis 2015;9:e3423.

35 Ndayishimiye O, Ortu G, Soares Magalhaes RJ, et al. Control of neglected tropical diseases in Burundi: partnerships, achievements, challenges, and lessons learned after four years of programme implementation. PLoS Negl Trop Dis 2014;8:e2684.
36 Mwingira UJ, Means AR, Chikawe M, et al. Integrating neglected tropical disease and immunization programs: the experiences of the Tanzanian Ministry of health. Am J Trop Med Hyg 2016;95:505-7.

37 Lankester F, Davis A, Kinung'hi S, et al. An integrated health delivery platform, targeting soil-transmitted helminths (STh) and canine mediated human rabies, results in cost savings and increased breadth of treatment for STh in remote communities in Tanzania. BMC Public Health 2019;19:1398.

38 Jacob BG, Loum D, Lakwo TL, et al. Community-directed vector control to supplement mass drug distribution for onchocerciasis elimination in the Madi mid-North focus of northern Uganda. PLOS Negl Trop Dis 2018;12:e0006702.

39 Okello WO, Okello AL, Inthavong P, et al. Improved methods to capture the total societal benefits of zoonotic disease control: demonstrating the cost-effectiveness of an integrated control programme for taenia solium, soil transmitted helminths and classical swine fever in northern Lao PDR. PLoS Negl Trop Dis 2018;12:1-22.

40 Sun L-P, Wang W, Zuo Y-P, et al. An integrated environmental improvement of marshlands: impact on control and elimination of schistosomiasis in marshland regions along the Yangtze river, China. Infect Dis Poverty 2017;6:1-10.

41 Chen Y-Y, Liu J-B, Huang X-B, et al. New integrated strategy emphasizing infection source control to curb schistosomiasis japonica in a marshland area of hubei Province, China: findings from an eight-year longitudinal survey. PLoS One 2014;9:e89779.

42 Page WA, Judd JA, MacLaren DJ, et al. Integrating testing for chronic strongyloidiasis within the Indigenous adult preventive health assessment system in endemic communities in the Northern Territory, Australia: an intervention study. PLoS Negl Trop Dis 2020;14:e0008232.

43 Hashimoto K, Zúniga C, Nakamura J, et al. Integrating an infectious disease programme into the primary health care service: a retrospective analysis of Chagas disease community-based surveillance in Honduras. BMC Health Serv Res 2015;15:116.

44 Rao PSSS, Gift N, Rao GS, et al. Elimination of leprosy: the integration of leprosy related activities into the general health services of Tamil Nadu. Lepr Rev 2002;73:123-9.

45 Kabatereine NB, Standley CJ, Sousa-Figueiredo JC, et al. Integrated prevalence mapping of schistosomiasis, soil-transmitted helminthiasis and malaria in lakeside and island communities in lake Victoria, Uganda. Parasit Vectors 2011;4:232.

46 Knipes AK, Frantz Lemoine J, Monestime F. Partnering for success: integrated transmission assessment surveys for lymphatic filariasis, soiltransmitted helminths and malaria in Haiti. Am J Trop Med Hyg 2015:93:375.

47 Midzi N, Mtapuri-Zinyowera S, Sangweme D, et al. Efficacy of integrated school based de-worming and prompt malaria treatment on helminths -Plasmodium falciparum co-infections: a 33 months follow up study. BMC Int Health Hum Rights 2011;11:9.

48 Knipes AK, Lemoine JF, Monestime F, et al. Partnering for impact: integrated transmission assessment surveys for lymphatic filariasis, soil transmitted helminths and malaria in Haiti. PLoS Negl Trop Dis 2017;11:e0005387

49 Amparo ACB, Mendoza ECB, Licuan DA, et al. Impact of integrating rabies education into the curriculum of public elementary schools in llocos Norte, Philippines on rabies knowledge, and animal bite incidence. Front Public Health 2019;7:1-11.

50 Mwinzi PNM, Montgomery SP, Owaga CO, et al. Integrated community-directed intervention for schistosomiasis and soil transmitted helminths in western Kenya - a pilot study. Parasit Vectors 2012:5:182.

51 Lemoine JF, Desormeaux AM, Monestime F, et al. Controlling neglected tropical diseases (NTDs) in Haiti: implementation strategies and evidence of their success. PLoS Negl Trop Dis 2016;10:e0004954.

52 Clarke NE, Clements ACA, Amaral S, et al. (S)WASH-D for Worms: A pilot study investigating the differential impact of school- versus community-based integrated control programs for soil-transmitted helminths. PLoS Negl Trop Dis 2018;12:1-18.

53 Emerson PM, Ngondi J, Biru E, et al. Integrating an NTD with one of "The big three": combined malaria and trachoma survey in Amhara Region of Ethiopia. PLoS Negl Trop Dis 2008;2:e197.

54 WHO. Evaluation of the WHO Neglected Tropical Diseases Programme Volume 1 [Internet], 2019. Available: https://www.sparc. bc.ca/wp-content/uploads/2016/12/continuum-of-collaboration.pdf

55 Hotez P, Raff S, Fenwick A, et al. Recent progress in integrated neglected tropical disease control. Trends Parasitol 2007;23:511-4.

56 Richards FO, Eigege A, Miri ES, et al. Integration of mass drug administration programmes in Nigeria: the challenge of schistosomiasis. Bull World Health Organ 2006;84:673-6. 
57 Molyneux DH, Nantulya VM. Linking disease control programmes in rural Africa: a pro-poor strategy to reach Abuja targets and millennium development goals. BMJ 2004;328:1129-32.

58 Ejike CU, Oluwole AS, Omitola OO, et al. Schisto and ladders version 2: a health educational board game to support compliance with school-based mass drug administration with praziquantel - a pilot study. Int Health 2021;13:281-90.

59 Parker M, Allen T. Does mass drug administration for the integrated treatment of neglected tropical diseases really work? assessing evidence for the control of schistosomiasis and soil-transmitted helminths in Uganda. Health Res Policy Syst 2011;9:3.

60 Baker MC, McFarland DA, Gonzales M, et al. The impact of integrating the elimination programme for lymphatic filariasis into primary health care in the Dominican Republic. Int $J$ Health Plann Manage 2007;22:337-52.

61 Rebollo MP, Sambou SM, Thomas B, et al. Elimination of lymphatic filariasis in the Gambia. PLoS Negl Trop Dis 2015;9:e0003642.

62 Molyneux D, Bush S, Bannerman R, et al. Neglected tropical diseases activities in Africa in the COVID-19 era: the need for a "hybrid" approach in COVID-endemic times. Infect Dis Poverty 2021;10:1-13.
63 Castro-Arroyave D, Monroy MC, Irurita MI. Integrated vector control of Chagas disease in Guatemala: a case of social innovation in health. Infect Dis Poverty 2020;9:25.

64 Gunawardena S, Gunawardena NK, Kahathuduwa G, et al. Integrated school-based surveillance for soil-transmitted helminth infections and lymphatic filariasis in Gampaha district, Sri Lanka. Am J Trop Med Hyg 2014;90:661-6.

65 Leslie J, Garba A, Boubacar K, et al. Neglected tropical diseases: comparison of the costs of integrated and vertical preventive chemotherapy treatment in niger. Int Health 2013;5:78-84.

66 Madon S, Malecela MN, Mashoto K, et al. The role of community participation for sustainable integrated neglected tropical diseases and water, sanitation and hygiene intervention programs: a pilot project in Tanzania. Soc Sci Med 2018;202:28-37.

67 Mani TR, Rajendran R, Sunish IP, et al. Effectiveness of two annual, single-dose mass drug administrations of diethylcarbamazine alone or in combination with albendazole on soil-transmitted helminthiasis in filariasis elimination programme. Trop Med Int Health 2004:9:1030-5. 